\title{
Diagnostic Performance of TE, 2D-SWE And MRE For Liver Fibrosis In Treatment-Naive People With HBV: A Systematic Review And Meta-Analysis
}

\author{
Mingkai Li \\ The Third Affiliated Hospital of Sun Yet-sun University Department of Gastroenterology \\ Sizhe Wan \\ The Third Affiliated Hospital of Sun Yet-sun University Department of Gastroenterology \\ Xiaoying Wu \\ The Third Affiliated Hospital of Sun Yet-sun University Department of Gastroenterology \\ Bin Wu ( $D$ wubin6@mail.sysu.edu.cn) \\ Third Affiliated Hospital of Sun Yat-Sen University https://orcid.org/0000-0001-9039-9681
}

\section{Research Article}

Keywords: Transient elastography, Two-dimensional shear wave elastography, Magnetic resonance elastography, Diagnostic performance, Treatment-naive, Hepatitis B, Liver, Liver fibrosis, Meta-analysis, Systematic review

Posted Date: July 27th, 2021

DOI: https://doi.org/10.21203/rs.3.rs-714753/v1

License: @ (i) This work is licensed under a Creative Commons Attribution 4.0 International License. Read Full License 


\section{Abstract}

Background/aims: To assess the performance of transient elastography (TE), two-dimensional shear wave elastography (2D-SWE), and magnetic resonance elastography (MRE) for staging significant fibrosis and cirrhosis in untreated chronic hepatitis B (CHB) patients.

Methods: Pubmed, Embase, Web of Science and Cochrane Library were searched for terms involving CHB, TE, SWE, and MRE. Other etiologies of chronic liver disease (CLD), previous treatment in patients or articles not published in SCl journals were excluded. Hierarchical non-linear models were used to evaluate the diagnostic accuracy of TE, 2D-SWE and MRE. Heterogeneity was explored via analysis of threshold effect and meta-regression.

Results: Twenty-eight articles with a total of 4540 untreated CHB patients were included. The summary AUROC using TE, 2D-SWE and MRE for predicting significant fibrosis (SF) were $0.84,0.89$, and 0.99 , respectively. MRE is more accurate than both TE $(P \otimes 0.01)$ and 2D-SWE (P凶0.01) in staging SF. 2D-SWE is superior to TE in detecting SF (Pख0.01). The summary AUROC employing TE, 2D-SWE and MRE for detecting cirrhosis were $0.9,0.94$, and 0.99 , respectively. TE displayed a similar diagnostic accuracy with 2D-SWE in staging cirrhosis $(P=0.14)$. MRE and 2D-SWE are comparable for staging cirrhosis $(P=0.08)$. MRE is superior than TE (Pख0.01) in staging cirrhosis.

Conclusion: TE, 2D-SWE, and MRE express acceptable diagnostic accuracies in staging staging significant fibrosis and cirrhosis in untreated CHB patients. Both MRE and 2D-SWE are better choices while the TE can be regarded as a secondary option.

\section{Introduction}

Despite the availability of effective drug interventions that reduce or prevent complications in most cases, chronic hepatitis $\mathrm{B}$ (CHB) remains a major public health issue worldwide that poses a significant health burden [1]. As the causative agent of CHB, human hepatitis B virus (HBV) is a hepatotropic DNA virus that can trigger acute and chronic hepatitis, contributing to HBV-related cirrhosis and even hepatocellular carcinoma [2]. Due to the lack of early signs in patients with HBV-related compensated cirrhosis, liver biopsy in the asymptomatic population is inadequate, and early diagnosis of HBV-related cirrhosis is not easy [3]. In the majority of cases, at the time of inpatient visits, the disease tends to progress to a decompensated period. Patients tend to suffer from various serious or even fatal complications, namely esophageal-gastric varices bleeding, refractory ascites, and hepatocellular carcinoma [4]. Several avenues point out that the progression of liver fibrosis can be halted or even reversed by early diagnosis, dynamic assessment and effective intervention that blocks persistent damage to the liver [5]. Hence, early detection of fibrosis and effective intervention of the related etiology are extremely fundamental to improve the prognosis of patients with chronic liver disease (CLD) [6].

Liver biopsy is the gold standard for assessing hepatitis and fibrosis. However, invasiveness, sampling error and inter-observer variations limit its clinical application [7]. More researchers have focused on noninvasive methods to more accurately assess liver fibrosis.

Imaging techniques such as transient elastography (TE), shear wave elastography (2D-SWE), and magnetic resonance elastography (MRE) have been proven to efficiently and precisely assess fibrosis efficiently and precisely in CHB patients. TE, also known as FibroScan, has been recommended by the World Health Organization for assessing fibrosis and has become widely present in clinical practice [8]. 2D-SWE is a well-validated device ultrasonographic elastography technique with various strengths such as offering a more precise region of interest (ROI) and monitoring blood flow alterations for high-quality measurements. Growing evidence has revealed that 2D-SWE is a favorable choice for staging fibrosis in CHB patients [9]. MRE is another elastography technique that utilizes a modified phase-contrast imaging sequence to estimate fibrosis via shear waves within the whole liver. Thus far, MRE has been considered the most accurate noninvasive method to assess liver fibrosis in CLD with great reliability and reproducibility [10].

These three imaging techniques have been proven to exhibit promising results for quantification of liver fibrosis with considerable accuracies. Dong et [11] concluded that MRE and 2D-SWE are excellent tools for staging fibrosis in patients with CHB. Nevertheless, they also included studies regardless of whether the patients were receiving antiviral therapy or not. Indeed, there is an urgent need to stage fibrosis for treatment-naive people with HBV. Given that CHB is a dynamic disease, persons who are not receiving treatment need be assessed regularly to determine whether to initiate antivirus treatment (AVT). If the biopsy specimen shows significant fibrosis (SF) in patients with elevated HBV DNA levels, AVT is recommended based on the American Association for the Study of Liver Diseases (AASLD) 2018 Hepatitis B Guidance [12].

The AASLD suggests that adults with compensated cirrhosis and low-level viremia $(<2,000 \mathrm{IU} / \mathrm{mL})$ be treated with antiviral therapy to reduce the risk of decompensation, regardless of ALT level [12]. To better tune the timing of AVT, we conduct a meta-analysis to compare the diagnostic performance of TE, 2DSWE, and MRE for staging SF and cirrhosis in treatment-naive people with HBV.

\section{Materials And Methods}

This study was conducted and reported according to the PRISMA (Preferred Reporting Items for Systematic Reviews and Meta-Analyses) guidelines [13]. The protocol was registered with PROSPERO (CRD42021248023).

\section{Articles search strategy}

The specific search strategy is listed in Supplementary Table 1. Four authorized online databases, namely Pubmed, Embase, the Web of Science, and the Cochrane Library (01/01/2000-01/02/2021) were screened utilizing the following words: hepatitis b, liver fibrosis, FibroScan, transient elastography, shear wave elastography and magnetic resonance elastography.

\section{Eligibility criteria}


The following situations were considered as the inclusion criteria: 1) the accuracies of 2D-SWE, TE or MRE for discriminating liver fibrosis in CHB patients were investigated; 2 ) the specific liver fibrosis stage of each patient was biopsy-proven; 3 ) the sensitivity, specificity and the number of patients in each fibrosis stage could be extracted to create $2 \times 2$ table of test performance; 4 ) at least 50 patients were enrolled in each investigation; and 5) the original articles need to be published in English and could be screened in SCl journals. The following situations were considered as the exclusion criteria: 1) the original articles did not focus on the diagnostic performance of TE, 2D-SWE or MRE; 2) special types of work such as patent, book section, case report, reply, letter, commentary, conference abstracts, review or meta-analysis were excluded; 3 ) studies on children or animal; 4) insufficient data to create $2 \times 2$ table of test performance; 5) patients were co-infected with other viral hepatitis or HIV; 6) patients were diagnosed as CLD triggered by other etiologies such as alcoholic liver disease (ALD), non-alcoholic fatty liver disease (NAFLD) and autoimmune liver disease; 7) patients had already received antiviral therapy, hepatectomy or liver transplant before biopsy or imaging tests; 8) patients were identified as hepatic carcinoma before TE, 2D-SWE, MRE, or liver biopsy; 9) unclear interval between imaging tests and liver biopsy or unclear liver biopsy size.

\section{Identification of liver fibrosis}

Significant fibrosis (SF) and cirrhosis were identified as stages F2-F4 and F4 using the corresponding scoring systems such as Scheuer, Ishak, Metavir, BattsLudwig, and Knodell.

\section{Data acquirement}

Two experienced researchers (ML and SW) were first invited to screen the online databases and make preliminary selections. The eligibility and quality of each article were screened by each investigator. Two researchers then extracted the targeted data separately. Basic characteristics, technical characteristics of the included studies as well as the diagnostic performance of these three noninvasive approaches were summarized in our predesigned forms.

\section{Quality assessment}

Quality Assessment of Diagnostic Accuracy Studies (QUADAS-2) tool was employed to conduct the evaluation of the quality of the included studies. The results of the QUADAS evaluation were visualized through Review Manager 5.3 (The Cochrane Collaboration). A third investigator (XW) was then invited to assess the discrepancies between the two researchers. The variation between the investigators were resolved through a discussion.

\section{Data synthesis and statistical analysis}

The demographic characteristic of the included patients were presented as mean \pm standard deviation (SD) or median \pm interquartile range. The number of true positives (tp), false positives ( $\mathrm{fp}$ ), false negatives ( $\mathrm{fn}$ ), and true negatives (tn) was calculated based on the reported population in each biopsy-proven fibrosis stage, sensitivity, and specificity of these three noninvasive imaging methods. Once the $2 \times 2$ table comprised of $t p, f p, f n$ and $t n$ was completed, the summary positive predictive value (PPV), negative predictive value (NPV), and area under the receiver operating characteristic curve (AUROC), positive likelihood ratio (LR) and negative LR were calculated according to the corresponding formulas. For meta-analysis, the pooled sensitivity and specificity were presented with midas and metannif modules in Stata 16.0 (StataCorp LP) [14]. The summary diagnostic odds ratios (DORs) were calculated utilizing a Der-Simonian and Laird random effects model with a corresponding test of heterogeneity. Hierarchical non-linear models including HSROC model and the bivariate model were used in our study to evaluate the diagnostic accuracy. The non-threshold heterogeneity was presented with the Q-12 statistic in the forest plots. An I-squared value $>50 \%$ was regarded as a threshold for determining substantial statistical heterogeneity [15]. SAS 9.4 (SAS Institute Inc) with a "mixed" command was utilized for the multiple comparisons of the sensitivity and the specificity among TE, 2D-SWE and MRE [16]. R 4.0.3 (R Foundation for Statistical Computing) with "mada" was utilized to compare the fitted SROC curves of TE, 2D-SWE, and MRE [17]. The pairwise comparisons of the AUROC values were conducted through the DeLong test [18]. A $P$ value $<0.05$ was considered to indicate statistically significant differences.

\section{Publication bias}

Deeks' funnel plots were generated by Stata 16.0 with "midas" command and a "mylabels" package for the evaluation of publication bias of the included studies. A $P<0.05$ was considered to indicate the existence of publication bias.

\section{Exploration of heterogeneity}

As different cut-off values were adopted in individual studies, the threshold effect was evaluated via spearman correlation analysis of the sensitivity and the specificity with MetaDisc 1.4. Meta-regression analysis were used to evaluate the influence of seven characteristics of individual studies on AUROC, namely the location of the study population (Asia vs Europe), study design (prospective cohort study vs retrospective cohort study or cross-sectional study), mean biopsy length ( $<20 \mathrm{~mm}$ vs $\geq 20 \mathrm{~mm}$ ), mean ALT ( $<5 \mathrm{ULN}$ vs $\geq 5 \mathrm{ULN}$ ), liver biopsies scoring system (Metavir vs non-Metavir), the interval between biopsy and imaging test ( $<3$ months vs $\geq 3$ months), study quality (all question score yes vs one or more questions scored no or unclear).

\section{Results}

\section{Characteristics and the Quality of the Retrieved Studies}

The flow diagram of the study selection is presented in Figure 1. 4190 records were retrieved utilizing our primary search strategies. 3609 articles were identified after duplications removed. After excluding the studies that did not fulfill the eligibility criteria, 28 studies were ultimately included, which were listed in the reference portion of Supporting Information. Table 1-2 offer the basic characteristics and the technical characteristics of the included studies. 4540 subjects (mean age, 42.3 years; $67.2 \%$ male) were included. Because of the high hepatitis B prevalence in China, the majority of the included studies were from 
Asia (90\%). In addition to 7 (25\%) retrospective studies, 21 (75\%) studies were based on prospective design. Regarding the QUADAS-2 score, 5 (17.9\%) studies scored 14 points. $16(57.1 \%), 6(21.4 \%)$ and $1(3.5 \%)$ study scored $13,12,11$, and 10 points, respectively.

Table 1 Basic characteristics of the Included Studies 


\begin{tabular}{|c|c|c|c|c|c|c|c|c|c|c|c|c|c|}
\hline Study id & Region & Imaging & Design & Center & Subject & $\begin{array}{l}\text { Study } \\
\text { time }\end{array}$ & $\begin{array}{l}\text { Mean } \\
\text { age } \\
\text { (y) }\end{array}$ & $\begin{array}{l}\text { Male } \\
(\%)\end{array}$ & $\begin{array}{l}\mathrm{BMI} \\
(\mathrm{kg} / \mathrm{m} 2)\end{array}$ & $\begin{array}{l}\text { ALT } \\
(\mathrm{U} / \mathrm{L})\end{array}$ & $\begin{array}{l}\text { ALT } \geq 5 \text { ULN } \\
\text { excluded }\end{array}$ & $\begin{array}{l}\mathrm{HBeAg} \\
(+)(\%)\end{array}$ & $\begin{array}{l}\mathrm{HBI} \\
(\mathrm{log} \\
\mathrm{IU} / \mathrm{r}\end{array}$ \\
\hline $\begin{array}{l}\text { Cardoso } \\
2011\end{array}$ & France & TE & $\begin{array}{l}\text { Cross } \\
\text { sectional }\end{array}$ & $\begin{array}{l}\text { Single } \\
\text { center }\end{array}$ & 202 & $\begin{array}{l}2006- \\
2008\end{array}$ & 49 & 60 & $\begin{array}{l}24.4 \pm \\
3.5^{\star}\end{array}$ & $\begin{array}{l}92(40- \\
112)+\end{array}$ & Yes & 24 & 4.9: \\
\hline $\begin{array}{l}\text { Gaia } \\
2011\end{array}$ & Italy & TE & Prospective & $\begin{array}{l}\text { Single } \\
\text { center }\end{array}$ & 70 & $\begin{array}{l}2007- \\
2009\end{array}$ & 44 & 71.4 & $\begin{array}{l}24.3 \\
(16.7- \\
33.1) \S\end{array}$ & $\begin{array}{l}70(13- \\
464) \S\end{array}$ & No & NA & Det \\
\hline $\begin{array}{l}\text { Hennedige } \\
2017\end{array}$ & Singapore & MRE & Retrospective & $\begin{array}{l}\text { Single } \\
\text { center }\end{array}$ & 63 & $\begin{array}{l}2009- \\
2012\end{array}$ & 50.1 & 69.8 & $\begin{array}{l}24.9 \pm \\
4^{\star}\end{array}$ & NA & No & NA & NA \\
\hline $\begin{array}{l}\text { Jekarl } \\
2018\end{array}$ & Korea & TE & Retrospective & $\begin{array}{l}\text { Single } \\
\text { center }\end{array}$ & 151 & $\begin{array}{l}2011- \\
2013\end{array}$ & 44.6 & 66.9 & NA & $\begin{array}{l}2.08 \pm \\
2.67 *\end{array}$ & Yes & NA & 6.4 \\
\hline $\begin{array}{l}\text { Leung } \\
2013\end{array}$ & China & $\begin{array}{l}\text { TE, } \\
\text { SWE }\end{array}$ & Prospective & $\begin{array}{l}\text { Single } \\
\text { center }\end{array}$ & 226 & $\begin{array}{l}2011- \\
2012\end{array}$ & 48.8 & 65 & $\begin{array}{l}24.2 \\
(21.6- \\
27.3) \dagger\end{array}$ & $\begin{array}{l}69 \\
(37.5- \\
105) \dagger\end{array}$ & No & 100 & 5.6 \\
\hline Li 2018 & China & TE & Retrospective & $\begin{array}{l}\text { Single } \\
\text { center }\end{array}$ & 118 & $\begin{array}{l}2013- \\
2015\end{array}$ & 37 & 62.8 & $\begin{array}{l}22.5 \\
(20.4- \\
24.2) \dagger\end{array}$ & $\begin{array}{l}39 \pm \\
16^{\star}\end{array}$ & Yes & 66 & $\begin{array}{l}5.4 \\
7.5)\end{array}$ \\
\hline Liu 2019 & China & SWE & Prospective & $\begin{array}{l}\text { Single } \\
\text { center }\end{array}$ & 123 & $\begin{array}{l}2016- \\
2018\end{array}$ & 36.3 & 49.6 & $\begin{array}{l}20.5 \pm \\
2.2^{\star}\end{array}$ & $\begin{array}{l}33.5 \pm \\
22.3^{\star}\end{array}$ & Yes & 60.2 & $\begin{array}{l}6.6: \\
8.0 !\end{array}$ \\
\hline Liu 2014 & China & TE & Prospective & $\begin{array}{l}\text { Single } \\
\text { center }\end{array}$ & 108 & $\begin{array}{l}2011- \\
2012\end{array}$ & 40.1 & 75 & $22 \pm 3^{*}$ & $\begin{array}{l}53.2(8- \\
270) \S\end{array}$ & No & NA & NA \\
\hline Park 2019 & $\begin{array}{l}\text { South } \\
\text { Korea }\end{array}$ & $\begin{array}{l}\text { SWE, } \\
\text { MRE }\end{array}$ & Retrospective & $\begin{array}{l}\text { Single } \\
\text { center }\end{array}$ & 63 & $\begin{array}{l}2013- \\
2018\end{array}$ & 50.8 & 58.7 & $\begin{array}{l}23.4 \pm \\
3.4^{*}\end{array}$ & $\begin{array}{l}44 \pm \\
20.8^{\star}\end{array}$ & Yes & 55.6 & $\begin{array}{l}5.7\{ \\
\pm 1 . \ell\end{array}$ \\
\hline Seo 2015 & $\begin{array}{l}\text { South } \\
\text { Korea }\end{array}$ & TE & Retrospective & $\begin{array}{l}15 \\
\text { Centers }\end{array}$ & 567 & $\begin{array}{l}2006- \\
2014\end{array}$ & 45 & 66.7 & $\begin{array}{l}23.8 \\
(22.1- \\
25.7) \dagger\end{array}$ & $\begin{array}{l}48(35- \\
56)+\end{array}$ & Yes & NA & NA \\
\hline Shi 2014 & China & MRE & Prospective & $\begin{array}{l}\text { Single } \\
\text { center }\end{array}$ & 113 & $\begin{array}{l}2012- \\
2013\end{array}$ & 42 & 42.5 & $\begin{array}{l}21.71 \\
(17.8- \\
32.6) \S\end{array}$ & NA & No & NA & Det \\
\hline $\begin{array}{l}\text { Trembling } \\
2014\end{array}$ & Italy & TE & Prospective & $\begin{array}{l}\text { Single } \\
\text { center }\end{array}$ & 182 & NA & 46 & 71 & NA & $\begin{array}{l}110.3 \pm \\
103.4^{\star}\end{array}$ & No & 29.1 & NA \\
\hline $\begin{array}{l}\text { Venkatesh } \\
2014\end{array}$ & Singapore & MRE & Prospective & $\begin{array}{l}\text { Single } \\
\text { center }\end{array}$ & 63 & $\begin{array}{l}2009- \\
2011\end{array}$ & 50 & 69.8 & $\begin{array}{l}24.82 \pm \\
3.97^{\star}\end{array}$ & $\begin{array}{l}71.75 \pm \\
101.8^{\star}\end{array}$ & No & NA & NA \\
\hline Yao 2020 & China & $\begin{array}{l}\text { TE, } \\
\text { SWE }\end{array}$ & Retrospective & $\begin{array}{l}\text { Single } \\
\text { center }\end{array}$ & 54 & $\begin{array}{l}2013- \\
2015\end{array}$ & 36.7 & 76 & $\begin{array}{l}23.9 \\
(21.9- \\
25) \dagger\end{array}$ & $\begin{array}{l}50.4 \\
(28.8- \\
129.2) \dagger\end{array}$ & No & 61 & $\begin{array}{l}4.8 \\
6.3)\end{array}$ \\
\hline $\begin{array}{l}\text { Zeng } \\
2017\end{array}$ & China & $\begin{array}{l}\text { TE, } \\
\text { SWE }\end{array}$ & Prospective & $\begin{array}{l}\text { Single } \\
\text { center }\end{array}$ & 257 & $\begin{array}{l}2013- \\
2015\end{array}$ & 36.7 & 77.4 & $\begin{array}{l}21.7 \\
(19.7- \\
23.9) \dagger\end{array}$ & $\begin{array}{l}42 \\
(28.3- \\
67.8) \dagger\end{array}$ & No & NA & NA \\
\hline $\begin{array}{l}\text { Zeng } \\
2014\end{array}$ & China & SWE & Prospective & $\begin{array}{l}\text { Single } \\
\text { center }\end{array}$ & 104 & $\begin{array}{l}2011- \\
2012\end{array}$ & 37.2 & 78.8 & $\begin{array}{l}22.1 \pm \\
3.4^{\star}\end{array}$ & $\begin{array}{l}43(28- \\
74) \dagger\end{array}$ & No & NA & Det \\
\hline $\begin{array}{l}\text { Zhang } \\
2015\end{array}$ & China & TE & Retrospective & $\begin{array}{l}\text { Single } \\
\text { center }\end{array}$ & 180 & $\begin{array}{l}2011- \\
2013\end{array}$ & 36.43 & 77.2 & $\begin{array}{l}24.36 \pm \\
3.52^{\star}\end{array}$ & $\begin{array}{l}93.88 \pm \\
116.82^{\star}\end{array}$ & No & NA & $\begin{array}{l}4.6 ! \\
2.6 !\end{array}$ \\
\hline $\begin{array}{l}\text { Osakabe } \\
2011\end{array}$ & Japan & TE & Prospective & $\begin{array}{l}\text { Single } \\
\text { center }\end{array}$ & 51 & $\begin{array}{l}2005- \\
2009\end{array}$ & 51 & 36.8 & NA & $\begin{array}{l}34 \\
(20.3- \\
80) \dagger\end{array}$ & No & 32.5 & 4.6 \\
\hline Cho 2011 & Korea & TE & Prospective & $\begin{array}{l}\text { Single } \\
\text { center }\end{array}$ & 121 & $\begin{array}{l}2006- \\
2009\end{array}$ & 39.1 & 66.9 & $\begin{array}{l}23.9 \pm \\
3.0^{*}\end{array}$ & $\begin{array}{l}167.0 \pm \\
197.9^{\star}\end{array}$ & No & NA & Det \\
\hline $\begin{array}{l}\text { Kim BK } \\
2012\end{array}$ & Korea & TE & Prospective & $\begin{array}{l}\text { Single } \\
\text { center }\end{array}$ & 194 & $\begin{array}{l}2008- \\
2010\end{array}$ & 46.7 & 61.3 & $\begin{array}{l}23.4 \pm \\
2.8^{*}\end{array}$ & $\begin{array}{l}58.4 \pm \\
27.1^{\star}\end{array}$ & No & NA & NA \\
\hline $\begin{array}{l}\text { Kim US } \\
2012\end{array}$ & Korea & TE & Prospective & $\begin{array}{l}\text { Single } \\
\text { center }\end{array}$ & 150 & $\begin{array}{l}2007- \\
2009\end{array}$ & 41.9 & 71.3 & $\begin{array}{l}23.2 \pm \\
2.8^{*}\end{array}$ & $\begin{array}{l}74.1 \pm \\
98.3^{*}\end{array}$ & No & NA & NA \\
\hline $\begin{array}{l}\text { Kumar } \\
2013\end{array}$ & India & TE & Prospective & $\begin{array}{l}\text { Single } \\
\text { center }\end{array}$ & 200 & $\begin{array}{l}2009- \\
2011\end{array}$ & 37.6 & 79.5 & $\begin{array}{l}24.2 \pm \\
3.7^{\star}\end{array}$ & $\begin{array}{l}44(9- \\
320) \S\end{array}$ & No & 40.5 & $\begin{array}{l}5.6 ! \\
(3.3 \\
8.8 !\end{array}$ \\
\hline $\begin{array}{l}\text { Zhao } \\
2017\end{array}$ & China & TE & Prospective & $\begin{array}{l}\text { Single } \\
\text { center }\end{array}$ & 99 & $\begin{array}{l}2014- \\
2015\end{array}$ & 37.7 & 64.6 & $\begin{array}{l}23.87 \pm \\
3.42^{\star}\end{array}$ & $\begin{array}{l}46.73 \pm \\
30.19^{\star}\end{array}$ & Yes & NA & NA \\
\hline $\begin{array}{l}\text { Shen } \\
2019\end{array}$ & China & TE & Prospective & $\begin{array}{l}\text { Single } \\
\text { center }\end{array}$ & 593 & $\begin{array}{l}2014- \\
2015\end{array}$ & 37 & 63.7 & NA & NA & Yes & 59.5 & NA \\
\hline
\end{tabular}




\begin{tabular}{|c|c|c|c|c|c|c|c|c|c|c|c|c|c|}
\hline $\begin{array}{l}\text { Kim Do } \\
2009\end{array}$ & Korea & TE & Prospective & $\begin{array}{l}\text { Single } \\
\text { center }\end{array}$ & 91 & $\begin{array}{l}2005- \\
2006\end{array}$ & 42.5 & 79.2 & $\begin{array}{l}25.3 \pm \\
1.3^{*}\end{array}$ & $\begin{array}{l}45.1 \pm \\
23.4^{*}\end{array}$ & No & 58.5 & NA \\
\hline $\begin{array}{l}\text { Kim US } \\
2009\end{array}$ & Korea & TE & Prospective & $\begin{array}{l}\text { Single } \\
\text { center }\end{array}$ & 130 & $\begin{array}{l}2006- \\
2007\end{array}$ & 40 & 80.2 & $\begin{array}{l}23.8 \pm \\
4.6 *\end{array}$ & $\begin{array}{l}46 \pm \\
24 *\end{array}$ & No & 59.3 & $\begin{array}{l}50 . ! \\
\text { dett }\end{array}$ \\
\hline $\begin{array}{l}\text { Chan } \\
2009\end{array}$ & China & TE & Prospective & $\begin{array}{l}\text { Single } \\
\text { center }\end{array}$ & 161 & $\begin{array}{l}2006- \\
2008\end{array}$ & 45 & 76 & $24 \pm 4^{\star}$ & $\begin{array}{l}93 \pm \\
78 *\end{array}$ & No & 43 & 6.5 \\
\hline Wu 2015 & China & MRE & Prospective & $\begin{array}{l}\text { Single } \\
\text { center }\end{array}$ & 106 & $\begin{array}{l}2011- \\
2013\end{array}$ & 59 & 48.7 & NA & NA & No & NA & NA \\
\hline
\end{tabular}

*Mean $\pm S D$.

†Meadian (interquartile range).

§Meadian (range).

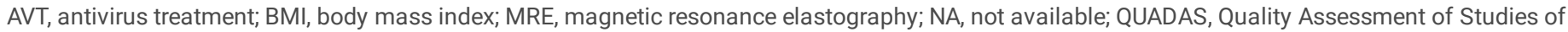
Diagnostic Accuracy Studies; SWE, shear wave elastography; TE, Transient Elastography; ULN, upper limit of normal.

Table 2 Technical characteristics of imaging and histological examination 


\begin{tabular}{|c|c|c|c|c|c|c|c|}
\hline Id & Author & year & Imaging & Instrument detail & Scoring system & Interval & Length \\
\hline 1 & Cardoso & 2011 & TE & M probe (1-6 MHz) & Metavir & ष1 day & $\geq 15 \mathrm{~mm}$ \\
\hline 2 & Gaia & 2011 & TE & M probe (1-6 MHz) & MEtavir & 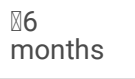 & $\geq 20 \mathrm{~mm}$ \\
\hline 3 & Hennedige & 2017 & MRE & 1.5T $(60 \mathrm{~Hz}) ; 2 \mathrm{D}-\mathrm{GRE}$ sequence & Metavir & 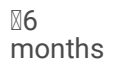 & $\geq 15 \mathrm{~mm}$ \\
\hline 4 & Jekarl & 2018 & TE & M probe (1-6 MHz) & Knodell & \$1 day & $\geq 20 \mathrm{~mm}$ \\
\hline 5 & Leung & 2013 & TE, SWE & M probe (1-6 MHz); SC6-1 probe (1-6 MHz) & Metavir & \1 year & $\geq 15 \mathrm{~mm}$ \\
\hline 6 & $\mathrm{Li}$ & 2018 & TE & M probe (1-6 MHz) & Metavir & 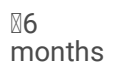 & $\geq 15 \mathrm{~mm}$ \\
\hline 7 & Liu & 2014 & TE & M probe (1-6 MHz) & Metavir & \$1 day & $\begin{array}{l}15-19 \\
\mathrm{~mm}\end{array}$ \\
\hline 8 & Liu & 2019 & SWE & SC6-1 probe (1-6 MHz) & Scheuer & ष1 day & $\geq 15 \mathrm{~mm}$ \\
\hline 9 & Park & 2019 & $\begin{array}{l}\text { SWE, } \\
\text { MRE }\end{array}$ & $\begin{array}{l}\text { SC6-1 probe (1-6 MHz); } 3 \mathrm{~T} \text { (unclear frenquency), unclear } \\
\text { sequence }\end{array}$ & Metavir & 『2weeks & $\geq 10 \mathrm{~mm}$ \\
\hline 10 & Seo & 2015 & TE & M probe (1-6 MHz) & Batts and Ludwig & 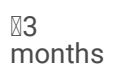 & $\begin{array}{l}15-30 \\
\mathrm{~mm}\end{array}$ \\
\hline 11 & Shi & 2014 & MRE & 1.5T (60 Hz); 2D-GRE sequence & Metavir & 23 days & $\begin{array}{l}14 \pm 7 \\
\mathrm{~mm}\end{array}$ \\
\hline 12 & Trembling & 2014 & TE & M probe (1-6 MHz) & Metavir & $\nabla 1$ day & $\geq 20 \mathrm{~mm}$ \\
\hline 13 & Venkatesh & 2014 & MRE & 1.5T $(60 \mathrm{~Hz}) ; 2 \mathrm{D}-\mathrm{GRE}$ sequence & Metavir & 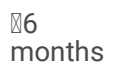 & $\geq 18 \mathrm{~mm}$ \\
\hline 14 & Yao & 2020 & TE, SWE & M probe (1-6 MHz); SC6-1 probe (1-6 MHz) & Ishak & $\begin{array}{l}\otimes 1 \\
\text { month }\end{array}$ & $\geq 20 \mathrm{~mm}$ \\
\hline 15 & Zeng & 2014 & SWE & SC6-1 probe (1-6 MHz) & Metavir & $\varangle 3$ days & $\geq 15 \mathrm{~mm}$ \\
\hline 16 & Zeng & 2017 & TE, SWE & M probe (1-6 MHz); SC6-1 probe (1-6 MHz) & Metavir & $\unrhd 3$ days & $\geq 15 \mathrm{~mm}$ \\
\hline 17 & Zhang & 2015 & TE & M probe (1-6 MHz) & Scheuer & \3 days & $\geq 15 \mathrm{~mm}$ \\
\hline 18 & Osakabe & 2011 & TE & M probe(1-6MHz) & Metavir & $<1$ month & $\geq 15 \mathrm{~mm}$ \\
\hline 19 & Cho & 2011 & TE & M probe(1-6MHz) & Metavir & $<1$ day & $\geq 15 \mathrm{~mm}$ \\
\hline 20 & Kim BK & 2012 & TE & M probe(1-6MHz) & $\begin{array}{l}\text { Batts and Ludwig scoring } \\
\text { system }\end{array}$ & $<1$ day & $\geq 20 \mathrm{~mm}$ \\
\hline 21 & Kim US & 2012 & TE & M probe(1-6MHz) & Laennec & $<1$ day & $\geq 15 \mathrm{~mm}$ \\
\hline 22 & Kumar & 2013 & TE & M probe(1-6MHz) & Metavir & $<1$ week & $\geq 15 \mathrm{~mm}$ \\
\hline 23 & Zhao & 2017 & TE & M probe(1-6MHz) & Metavir & $<1$ day & $\geq 15 \mathrm{~mm}$ \\
\hline 24 & Shen & 2019 & TE & M probe(1-6MHz) & Metavir & $<1$ week & $\geq 15 \mathrm{~mm}$ \\
\hline 25 & Kim Do & 2009 & TE & M probe(1-6MHz) & Metavir & $<1$ day & $\geq 10 \mathrm{~mm}$ \\
\hline 26 & Kim US & 2009 & TE & M probe(1-6MHz) & Metavir & $<1$ day & $\geq 15 \mathrm{~mm}$ \\
\hline 27 & Chan & 2009 & TE & M probe(1-6MHz) & Metavir & $<1$ month & $\geq 15 \mathrm{~mm}$ \\
\hline 28 & Wu & 2015 & MRE & $1.5 \mathrm{~T}(60 \mathrm{~Hz}) ; 2 \mathrm{D}-\mathrm{GRE}$ sequence & Metavir & 3months & $\geq 10 \mathrm{~mm}$ \\
\hline
\end{tabular}

MRE, magnetic resonance elastography; SWE, shear wave elastography; TE, Transient Elastography.

\section{Diagnosing SF}

Twenty-three studies (3879 untreated patients) focused on the diagnostic performance of TE, 2D-SWE, and MRE for staging SF. Specifically, 16 (3244 patients), 6 (827 patients), and 5 (408 patients) studies investigated the TE, 2D-SWE, and MRE.

Table 3 demonstrates the diagnostic performance of these three methods for staging SF. The pooled sensitivity and specificity of TE were 0.76 ( $95 \% \mathrm{Cl}, 0.72$ 0.79 ) and $0.79(95 \% \mathrm{Cl}, 0.75-0.83)$, respectively. As shown in Figure 2, the summary AUROC of TE was $0.84(95 \% \mathrm{Cl}, 0.81-0.87)$. Regarding the $2 \mathrm{D}-\mathrm{SWE}$, the pooled sensitivity and specificity were $0.84(95 \% \mathrm{Cl}, 0.8-0.88)$ and $0.84(95 \% \mathrm{Cl}, 0.76-0.89)$. The AUROC was 0.89 (95\% Cl, $0.86-0.92)$. The overall diagnostic performance of MRE is $95 \%$ sensitivity, $96 \%$ specificity and $99 \%$ accuracy, at cut-off values ranged from 2.47 to $4.07 \mathrm{kpa}$.

Table 3 Summary sensitivity, specificity, PLR, NLR, DOR and AUROC of TE, SWE and MRE for staging fibrosis by bivariate analysis 


\begin{tabular}{|c|c|c|c|c|c|c|c|}
\hline $\begin{array}{l}\text { Imaging } \\
\text { test }\end{array}$ & $\begin{array}{l}\text { No. of studies (No. of } \\
\text { patients) }\end{array}$ & $\begin{array}{l}\text { Cut-off } \\
\text { Values }\end{array}$ & $\begin{array}{l}\text { Sensitivity }(95 \% \\
\mathrm{Cl})\end{array}$ & $\begin{array}{l}\text { Specificity (95\% } \\
\mathrm{Cl})\end{array}$ & PLR (95\% Cl) & NLR $(95 \% \mathrm{Cl})$ & $\begin{array}{l}\text { AUROC }(95 \% \\
\mathrm{CI})\end{array}$ \\
\hline \multicolumn{8}{|c|}{ Staging SF } \\
\hline TE & $16(3244)$ & $6-8.8$ & $0.76(0.72-0.79)$ & $0.79(0.75-0.83)$ & $3.65(3.08-4.32)$ & $\begin{array}{l}0.31(0.26- \\
0.36)\end{array}$ & $\begin{array}{l}0.84(0.81- \\
0.87)\end{array}$ \\
\hline SWE & $6(827)$ & 6.73-9.05 & $0.84(0.8-0.88)$ & $0.84(0.76-0.89)$ & $5.15(3.47-7.66)$ & $\begin{array}{l}0.19(0.15- \\
0.24)\end{array}$ & $\begin{array}{l}0.89(0.86- \\
0.92)\end{array}$ \\
\hline MRE & $5(408)$ & $2.47-4.07$ & $0.95(0.88-0.98)$ & $0.96(0.91-0.98)$ & $\begin{array}{l}24.94(10.14- \\
61.3)\end{array}$ & $\begin{array}{l}0.06(0.02- \\
0.13)\end{array}$ & $\begin{array}{l}0.99(0.97- \\
0.99)\end{array}$ \\
\hline \multicolumn{8}{|c|}{ Staging Cirrhosis } \\
\hline TE & 19 (3806) & 8-14.1 & $0.84(0.78-0.88)$ & $0.84(0.8-0.88)$ & $5.1(4.13-6.3)$ & $\begin{array}{l}0.19(0.14- \\
0.26)\end{array}$ & $\begin{array}{l}0.9(0.88- \\
0.93)\end{array}$ \\
\hline SWE & $5(773)$ & $9.5-11.8$ & $0.91(0.82-0.96)$ & $0.89(0.84-0.92)$ & $7.97(5.61-11.32)$ & $\begin{array}{l}0.1(0.04- \\
0.21)\end{array}$ & $\begin{array}{l}0.94(0.92- \\
0.96)\end{array}$ \\
\hline MRE & $5(408)$ & $3.46-6.87$ & $0.96(0.85-0.99)$ & $0.96(0.92-0.98)$ & $\begin{array}{l}25.68(11.28- \\
58.47)\end{array}$ & $\begin{array}{l}0.04(0.01- \\
0.16)\end{array}$ & $0.99(0.98-1)$ \\
\hline
\end{tabular}

AUROC, area under summary receiver operating characteristic; $\mathrm{Cl}$, confidence interval; DOR, diagnostic odds ratio; MRE, magnetic resonance elastography; PLR, positive likelihood ratio; NLR, negative likelihood ratio; SF, significant fibrosis; SWE, shear wave elastography; TE, transient elastography.

Pairwise comparisons was shown in Table 4. MRE expressed higher sensitivity than both TE $(\mathrm{F}=18.89, P \otimes 0.01)$ and 2D-SWE (F=6.13, $P \otimes 0.01)$. MRE exhibited greater specificity than both TE $(\mathrm{F}=13.67, P \otimes 0.01)$ and 2D-SWE $(\mathrm{F}=8.83, P \otimes 0.01)$. Compared with $\mathrm{TE}, 2 \mathrm{D}-\mathrm{SWE}$ displayed greater sensitivity $(\mathrm{F}=5.57, P=0.02)$. The specificity of TE and 2D-SWE were comparable $(F=0.92, P=0.34)$. As presented in Supplementary Figure 2 and Table 4, MRE was more accurate than both TE $(Z=8.88, P \otimes 0.01)$ and 2D-SWE (Z=5.16, $P \otimes 0.01)$. 2D-SWE was superior to TE $(Z=3.53, P \otimes 0.01)$. As shown in Figure 3 , in descending order, the combined DORs of MRE, 2D-SWE, and TE for staging SF were $299.18\left(I^{2}=0, P=0.565\right), 26.3\left(I^{2}=30.5 \%, P=0.207\right), 11.59\left(I^{2}=49.5 \%, P=0.013\right)$.

Table 4 Pairwise comparison of the pooled sensitivity, specificity and AUROC values of TE, SWE, and MRE for staging fibrosis

\begin{tabular}{|c|c|c|c|c|c|c|c|}
\hline \multirow{2}{*}{$\begin{array}{l}\text { Staging } \\
\text { fibrosis }\end{array}$} & \multirow{2}{*}{$\begin{array}{l}\text { Pairwise } \\
\text { comparisons }\end{array}$} & \multicolumn{2}{|c|}{ Sensitivity } & \multicolumn{2}{|c|}{ Specificity } & \multicolumn{2}{|l|}{ AUROC } \\
\hline & & $F$ value & $P$ value & F value & $P$ value & $Z$ value & $P$ value \\
\hline \multirow[t]{3}{*}{ Staging SF } & TE vs. SWE & 5.57 & 0.02 & 0.92 & 0.34 & 3.53 & $\varangle 0.01$ \\
\hline & SWE vs. MRE & 6.13 & 0.01 & 8.83 & $\nabla 0.01$ & 5.16 & $\nabla 0.01$ \\
\hline & TE vs. MRE & 18.89 & $\otimes 0.01$ & 13.67 & $\varangle 0.01$ & 8.88 & $\varangle 0.01$ \\
\hline \multirow[t]{3}{*}{ Staging cirrhosis } & TE vs. SWE & 2.35 & 0.13 & 3.04 & 0.08 & 1.47 & 0.14 \\
\hline & SWE vs. MRE & 0.37 & 0.55 & 3.92 & 0.04 & 1.73 & 0.08 \\
\hline & TE vs. MRE & 4.06 & 0.04 & 12.4 & $\triangle 0.01$ & 4.71 & $\triangle 0.01$ \\
\hline
\end{tabular}

AUROC, area under summary receiver operating characteristic; MRE, magnetic resonance elastography; SF, significant fibrosis; SWE, shear wave elastography; $\mathrm{TE}$, transient elastography.

\section{Diagnosing Cirrhosis}

Twenty-six studies (with 4441 treatment-naive CHB patients) investigated these three noninvasive methods for the prediction of cirrhosis. 19 (3806 patients), 5 (773 patients), and 5 (408 patients) items focused on the TE, 2D-SWE, and MRE, respectively for diagnosing cirrhosis.

Table 3 summarizes the diagnostic performance of these three methods for staging cirrhosis. The pooled sensitivity and specificity of TE were 0.84 ( $95 \% \mathrm{Cl}$, 0.78-0.88) and $0.84(95 \% \mathrm{Cl}, 0.8-0.88)$. As shown in Figure 2, the summary AUROC of TE was $0.9(95 \% \mathrm{Cl}, 0.88-0.93)$. Regarding the 2D-SWE, the pooled sensitivity and specificity were $0.91(95 \% \mathrm{Cl}, 0.82-0.96)$ and $0.89(95 \% \mathrm{Cl}, 0.84-0.92)$. The AUROC of 2D-SWE was $0.94(95 \% \mathrm{Cl}, 0.92-0.96)$. The overall diagnostic performance of MRE is $96 \%$ sensitivity, $96 \%$ specificity and $99 \%$ accuracy, at cut-off values ranged from 3.46 to $6.87 \mathrm{kpa}$.

Pairwise comparisons were shown in Table 4. MRE expressed higher sensitivity than MRE $(F=4.06, P=0.04)$. MRE expressed similar sensitivity with 2D-SWE $(F=0.37, P=0.55)$. MRE expressed higher specificity than both TE $(F=12.4, P \otimes 0.01)$ and 2D-SWE $(F=3.92, P=0.04)$. Compared with TE, 2D-SWE displayed similar sensitivity with TE $(F=2.53, P=0.13)$. The specificity of $2 D-S W E$ was comparable to that of $T E(F=3.04, P=0.08)$. As presented in Supplementary Figure 2 and Table 4, MRE was more accurate than TE $(Z=4.71, P \otimes 0.01)$. MRE displayed similar accuracy with $S W E(Z=1.73, P=0.08)$. The accuracies of 2D-SWE and TE were comparable $(\mathrm{F}=1.47, P=0.14)$. As shown in Figure 4, in descending order, the combined DORs of MRE, 2D-SWE, and TE for staging cirrhosis were 223.9 $\left(I^{2}=0, P=0.607\right), 70.62\left(I^{2}=59.6 \%, P=0.042\right)$ and $24.21\left(I^{2}=58.7 \%, P=0.001\right)$.

\section{Heterogeneity and Publication Bias}


Non-threshold heterogeneity was observed in TE, 2D-SWE and MRE for detecting significant fibrosis and cirrhosis (Supplementary Table 3). A meta-regression analysis can only be conducted in groups of more than 10 studies with complete data to examine the methodological heterogeneity. In groups larger than 10 studies, heterogeneity existed when TE was used for staging fibrosis. The diagnostic accuracy was not affected by the following factors when TE was used for staging significant fibrosis and cirrhosis: study design $(P=0.18$ and 0.87$)$, classification criteria $(P=0.5$ and 0.21$)$, region $(P=0.4$ and 0.49$)$, interval between biopsy and imaging test $(P=0.55$ and 0.32$)$, obviously abnormal ALT $(P=0.9$ and 0.94$)$, liver biopsy length $(P=0.33$ and 0.71$)$, and QUADAS-2 score $(P=0.1$ and 0.94$)$. There was no evidence of publication bias for TE, 2D-SWE and MRE for staging fibrosis (Supplementary Figure 3 ).

\section{Discussion}

In this meta-analysis, we evaluated the diagnostic performance of TE, 2D-SWE and MRE for staging fibrosis in CHB. This study demonstrates that TE, 2D-SWE, and MRE express acceptable diagnostic accuracies to stage significant fibrosis and cirrhosis in treatment-naive people with HBV. Additionally, for staging SF and cirrhosis, both MRE and 2D-SWE are both the best choices while TE can be regarded as a secondary option. For staging SF, the summary AUROC value of MRE is significantly greater than that of $2 \mathrm{D}-\mathrm{SWE}(0.99$ vs $0.89, P<0.01)$ and TE $(0.99$ vs $0.84, P<0.01)$. 2D-SWE is superior to TE (AUROC $=0.89$ vs $0.84, P<$ 0.01). For discriminating cirrhosis, MRE is superior to TE (AUROC $=0.99$ vs $0.9, P<0.01$ ), but comparable to $2 \mathrm{D}-\mathrm{SWE}(\mathrm{AUROC}=0.99 \mathrm{vs} 0.9, P=0.08$ ). Moreover, the diagnostic accuracy of MRE for staging SF and cirrhosis was both greater than 0.95 using AUROC, at cut-off values $2.47-4.07 \mathrm{kpa}$ and $3.46-6.87 \mathrm{kpa}$. MRE expressed the best diagnostic performance for staging SF and cirrhosis, which is also consistent with previous findings in patients with CLD [19; 20]. For discriminating cirrhosis in patients with other etiologies such as NAFLD, MRE and 2D-SWE were also comparable. Both MRE and 2D-SWE were regarded as better choices for staging cirrhosis [21].

Venkatesh et al. [22] confirmed that normal LSM assessed through MRE in normal Asian population is highly reproducible. The results were not affected by age, sex and body mass index (BMI). MRE can visualize the whole substantive organ without an accurate acoustic window, which is superior to TE [23]. A larger measurement area of the liver can effectively lower the sampling errors [24]. Ichikawa et al. [25] explained that TE can only conduct a unidirectional measurement, which is more likely to be interfered with reflection wave and refraction wave. In terms of MRE, it evaluates the two-dimensional (2D) or even three-dimensional (3D) displacement vector. Additionally, compared with TE, MRE can generate better quality of figures with compressional and continuous waves. Because MRE conducted with gradient-recalled echo (GRE) sequence has been well-validated in previous large cohorts of clinical studies [26], the most commonly applied commercial MRE technique is GRE-MRE [27]. Nevertheless, the conventional GRE-MRE technique tend to be technically deficient as the process of its imaging is easily susceptible to the iron deposition. Hence, GRE-MRE is rather time-consuming and a more stringent breath hold by the patients is required [28]. To lower these barriers, spin-echo-based echo planar imaging (SE-EPI) MRE sequence was developed. This novel sequence is less sensitive to the iron-overload and thus contributing to shorter imaging time and a higher technical success rate [29]. Despite the promising advances, MRE is currently time-consuming and costly. Considering the high prevalence of $\mathrm{CHB}$ and the scarcity of MRE in Asian countries, there is still a long way to popularize MRE for CHB patients on a large scale.

Compared with TE, easier access to the ROI with high quality measurements with a colorful elasticity map would be accessible through 2D-SWE [30]. Moreover, the variation of blood flow can be monitored through 2D-SWE [31; 32]. As inspired by its advantages and indicated by the results in our article, 2DSWE may be a better choice to stage SF than TE.

There are still limitations in this study. First, due to the incomplete data, our meta-regression analysis did not include the factors such as obesity, ascites, HBVDNA, which may also be the sources of heterogeneity and thus affecting our ultimate conclusions [33]. Surprisingly, Petzold et al. [34] pointed out that parameters such as age, gender, BMI, and liver function indexes had no significant impact on LSM measured by 2D-SWE. It is worth noting that the LSM measured through TE tends to be affected by inflammation, congestion, and cholestasis [35] and thus affecting our judgments. Second, this study did not take the financial cost, the convenience or success rate of examination into consideration. A cheaper and less time-consuming technique would lower the barrier for clinical applications [36]. Moreover, regarding 2D-SWE and MRE, although AUROCs are high, the number of studies on which rely these findings is rather small ( $n=6$ and 827 patients; $n=5$ and 408 patients), limiting the persuasiveness of our conclusions. Therefore, more prospective and multicenter studies are needed.

Collectively, our current study confirms that TE, 2D-SWE, and MRE express acceptable diagnostic accuracies in staging fibrosis in treatment-naïve people with HBV. MRE and 2D-SWE are both the best choices while TE can be regarded as a secondary option to stage SF and cirrhosis in untreated CHB patients.

\section{Abbreviations}

AASLD, American Association for the Study of Liver Diseases; AUROC, area under the receiver operating characteristic curve; AVT, antivirus treatment ; CHB, chronic hepatitis B; DOR, diagnostic odds ratio; HBV, hepatitis B virus; LR, likelihood ratio; MRE, magnetic resonance elastography; NAFLD, nonalcoholic fatty liver disease; NPV, negative predictive value; PPV, positive predictive value; QUADAS, quality assessment of diagnostic accuracy studies; ROC, receiver operating characteristic; SF, significant fibrosis; sROC: summary receiver operating characteristic; 2D-SWE, two-dimensional shear wave elastography; TE, transient elastography.

\section{Declarations}

Acknowledgements The authors would like to thank all the staff at the Library in the Third Affiliated Hospital of Sun Yat-Sen University for their great support and conscientious work during the literature search of this study.

Author contributions MKL, XYW and BW contributed to the literature search and data extraction. MKL and XYW conducted the study quality assessment. MKL and XYW performed the study design and data analysis. MKL drafted the manuscript. XYW and BW made the critical revision of the paper. BW contributed to 
the study conception and study supervision.

Funding This study has received funding by the National Natural Science Foundation of China (82070574), the Natural Science Foundation Team Project of Guangdong Province (2018B030312009), the Fundamental Research Funds for the Central Universities (19ykpy29).

\section{Compliance with ethical standards}

Conflict of interest The authors of this manuscript declare no relationships with any companies, whose products or services may be related to the subject matter of the article.

Ethical approval This article does not contain any studies with human participants or animals performed by any of the authors.

Informed consent Not applicable.

Clinical trials registration Not applicable.

Data sharing We agree with the policy in the journal.

\section{References}

1 Lau K, Burak K, Coffin C (2020) Impact of Hepatitis B Virus Genetic Variation, Integration, and Lymphotropism in Antiviral Treatment and Oncogenesis. Microorganisms 8

2 Liao Y (2020) Obstacles and opportunities in the prevention and treatment of HBV-related hepatocellular carcinoma. Genes \& diseases 7:291-298

3 Kramvis A, Kostaki E, Hatzakis A, Paraskevis D (2018) Immunomodulatory Function of HBeAg Related to Short-Sighted Evolution, Transmissibility, and Clinical Manifestation of Hepatitis B Virus. Frontiers in microbiology 9:2521

4 Fukui H, Saito H, Ueno Y et al (2016) Evidence-based clinical practice guidelines for liver cirrhosis 2015. Journal of gastroenterology 51:629-650

5 Yang Z, Peng Y, Yang S (2019) MicroRNA-146a regulates the transformation from liver fibrosis to cirrhosis in patients with hepatitis B via interleukin-6. Experimental and therapeutic medicine 17:4670-4676

6 Su C, Yang Y, Lin H (2018) Impact of etiological treatment on prognosis. Hepatology international 12:56-67

7 Johnson K, Laoveeravat P, Yee E, Perisetti A, Thandassery R, Tharian B (2020) Endoscopic ultrasound guided liver biopsy: Recent evidence. World journal of gastrointestinal endoscopy 12:83-97

8 Aberra H, Desalegn H, Berhe N et al (2019) The WHO guidelines for chronic hepatitis B fail to detect half of the patients in need of treatment in Ethiopia. Journal of hepatology 70:1065-1071

9 Chen S, Jiang T (2020) Preoperative noninvasive assessment for liver fibrosis in hepatocellular carcinoma patients with chronic hepatitis B: Comparison of two-dimensional shear-wave elastography with serum liver fibrosis models. European journal of radiology 133:109386

10 Zhang Y, Fowler K, Ozturk A et al (2020) Liver fibrosis imaging: A clinical review of ultrasound and magnetic resonance elastography. Journal of magnetic resonance imaging : JMRI 51:25-42

11 Dong Bingtian,Lyu Guorong,Chen Yuping et al. Comparison of two-dimensional shear wave elastography, magnetic resonance elastography, and three serum markers for diagnosing fibrosis in patients with chronic hepatitis B: a meta-analysis.[J] .Expert Rev Gastroenterol Hepatol, 2021, undefined: 1-13.

12 Terrault Norah A,Lok Anna S F,McMahon Brian J et al. Update on prevention, diagnosis, and treatment of chronic hepatitis B: AASLD 2018 hepatitis B guidance.[J] .Hepatology, 2018, 67: 1560-1599.

13 Moher D, Liberati A, Tetzlaff J, Altman D (2009) Preferred reporting items for systematic reviews and meta-analyses: the PRISMA statement. Journal of clinical epidemiology 62:1006-1012

14 Chang W, Peng F, Meng S, Xu J, Yang Y (2020) Diagnostic value of serum soluble triggering expressed receptor on myeloid cells 1 (sTREM-1) in suspected sepsis: a meta-analysis. BMC immunology $21: 2$

15 Groenendijk I, den Boeft L, van Loon L, de Groot L (2019) High Versus low Dietary Protein Intake and Bone Health in Older Adults: a Systematic Review and Meta-Analysis. Computational and structural biotechnology journal 17:1101-1112

16 Reitsma J, Glas A, Rutjes A, Scholten R, Bossuyt P, Zwinderman A (2005) Bivariate analysis of sensitivity and specificity produces informative summary measures in diagnostic reviews. Journal of clinical epidemiology 58:982-990

17 Dai N, Zhang X, Zhang Y et al (2016) Enhanced diagnostic utility achieved by myocardial blood analysis: A meta-analysis of noninvasive cardiac imaging in the detection of functional coronary artery disease. International journal of cardiology 221:665-673 
18 DeLong E, DeLong D, Clarke-Pearson D (1988) Comparing the areas under two or more correlated receiver operating characteristic curves: a nonparametric approach. Biometrics 44:837-845

19 Lefebvre T, Wartelle-Bladou C, Wong P et al (2019) Prospective comparison of transient, point shear wave, and magnetic resonance elastography for staging liver fibrosis. European radiology 29:6477-6488

20 Xiao H, Shi M, Xie Y, Chi X (2017) Comparison of diagnostic accuracy of magnetic resonance elastography and Fibroscan for detecting liver fibrosis in chronic hepatitis B patients: A systematic review and meta-analysis. PloS one 12:e0186660

21 Xiao G, Zhu S, Xiao X, Yan L, Yang J, Wu G (2017) Comparison of laboratory tests, ultrasound, or magnetic resonance elastography to detect fibrosis in patients with nonalcoholic fatty liver disease: A meta-analysis. Hepatology (Baltimore, Md) 66:1486-1501

22 Venkatesh S, Wang G, Teo L, Ang B (2014) Magnetic resonance elastography of liver in healthy Asians: normal liver stiffness quantification and reproducibility assessment. Journal of magnetic resonance imaging : JMRI 39:1-8

23 Abe H, Midorikawa Y, Okada M, Takayama T (2018) Clinical application of magnetic resonance elastography in chronic liver disease. Hepatology research : the official journal of the Japan Society of Hepatology 48:780-787

24 Huwart L, Sempoux C, Vicaut E et al (2008) Magnetic resonance elastography for the noninvasive staging of liver fibrosis. Gastroenterology 135:32-40

25 Ichikawa S, Motosugi U, Morisaka $\mathrm{H}$ et al (2015) Comparison of the diagnostic accuracies of magnetic resonance elastography and transient elastography for hepatic fibrosis. Magnetic resonance imaging 33:26-30

26 Loomba R, Wolfson T, Ang B et al (2014) Magnetic resonance elastography predicts advanced fibrosis in patients with nonalcoholic fatty liver disease: a prospective study. Hepatology (Baltimore, Md) 60:1920-1928

27 Hoodeshenas S, Yin M, Venkatesh S (2018) Magnetic Resonance Elastography of Liver: Current Update. Topics in magnetic resonance imaging : TMRI 27:319-333

28 Mariappan Y, Dzyubak B, Glaser K et al (2017) Application of Modified Spin-Echo-based Sequences for Hepatic MR Elastography: Evaluation, Comparison with the Conventional Gradient-Echo Sequence, and Preliminary Clinical Experience. Radiology 282:390-398

29 Choi S, Lee E, Ko A et al (2020) Technical success rates and reliability of spin-echo echo-planar imaging (SE-EPI) MR elastography in patients with chronic liver disease or liver cirrhosis. European radiology 30:1730-1737

30 Barr R, Ferraioli G, Palmeri M et al (2015) Elastography Assessment of Liver Fibrosis: Society of Radiologists in Ultrasound Consensus Conference Statement. Radiology 276:845-861

31 Dietrich C, Bamber J, Berzigotti A et al (2017) EFSUMB Guidelines and Recommendations on the Clinical Use of Liver Ultrasound Elastography, Update 2017 (Long Version). Ultraschall in der Medizin (Stuttgart, Germany : 1980) 38:e16-e47

32 Lok A, Perrillo R, Lalama C et al (2020) medLow Incidence of Adverse Outcomes in Adults with Chronic Hepatitis B Virus Infection in the Era of Antiviral Therapy. Hepatology (Baltimore, Md). 10.1002/hep.31554

33 Castera L (2014) Hepatitis B: are non-invasive markers of liver fibrosis reliable? Liver international : official journal of the International Association for the Study of the Liver. 10.1111/liv.12393:91-96

34 Petzold G, Bremer S, Knoop R et al (2020) Noninvasive assessment of liver fibrosis in a real-world cohort of patients with known or suspected chronic liver disease using 2D-shear wave elastography. European journal of gastroenterology \& hepatology 32:1559-1565

35 Oeda S, Tanaka K, Oshima A, Matsumoto Y, Sueoka E, Takahashi H (2020) Diagnostic Accuracy of FibroScan and Factors Affecting Measurements. Diagnostics (Basel, Switzerland) 10

36 Imajo K, Kessoku T, Honda Y et al (2016) Magnetic Resonance Imaging More Accurately Classifies Steatosis and Fibrosis in Patients With Nonalcoholic Fatty Liver Disease Than Transient Elastography. Gastroenterology 150:626-637.e627

\section{Figures}




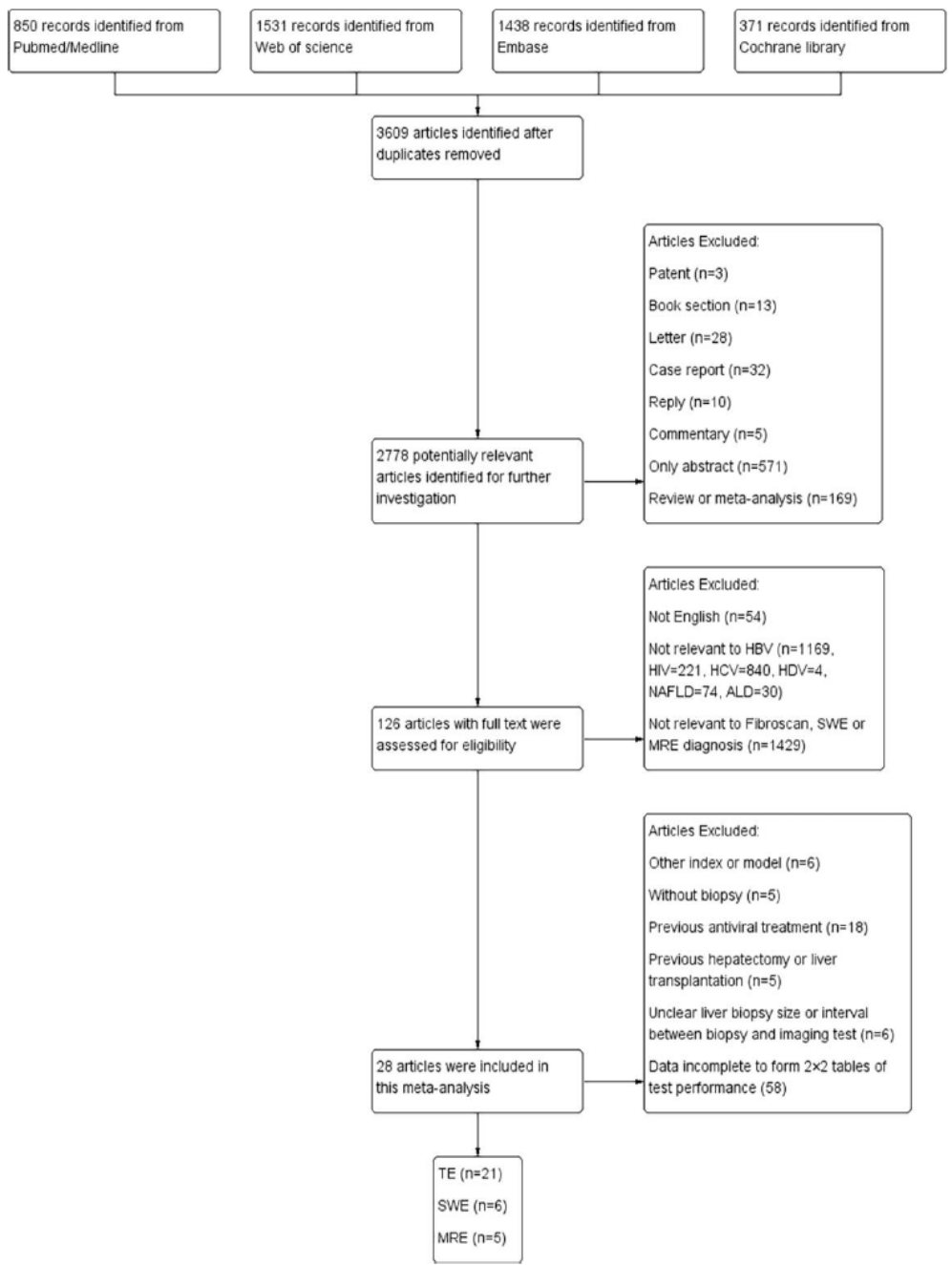

\section{Figure 1}

Flow diagram of the study selection. $150 \times 200 \mathrm{~mm}(300 \times 300 \mathrm{DPI})$ 


\section{Staging significant fibrosis:}

A

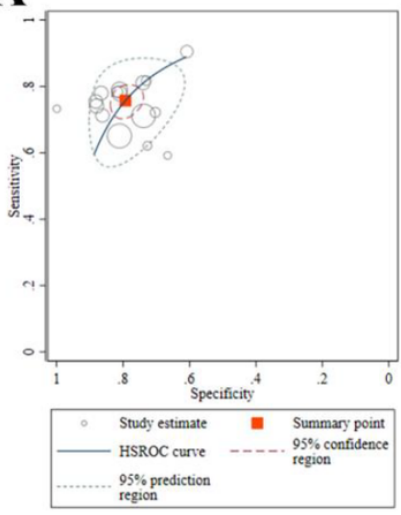

\section{Staging cirrhosis:}

D

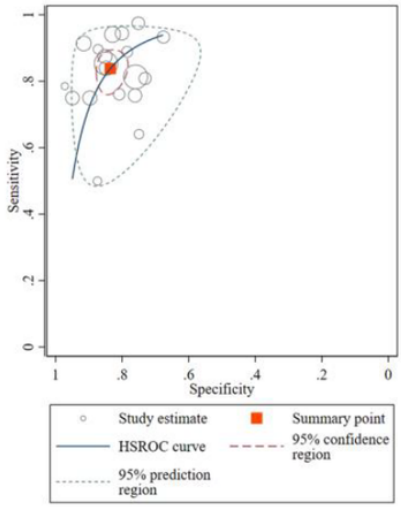

B

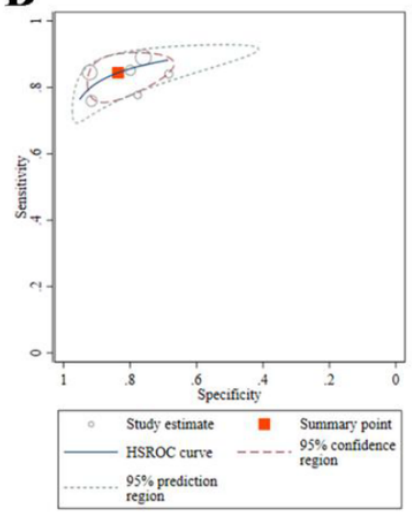

C

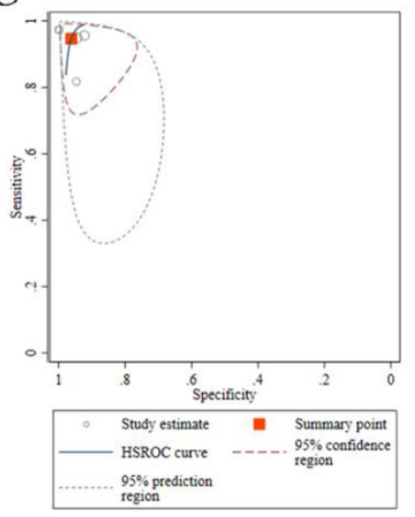

E

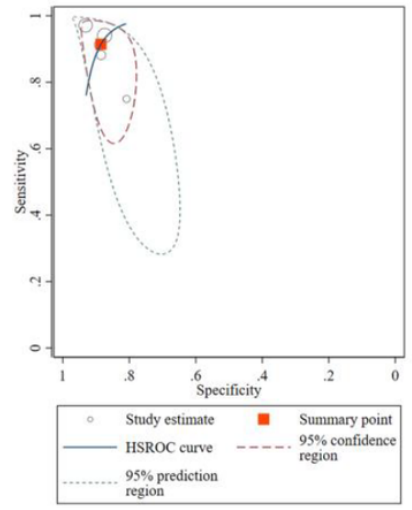

F

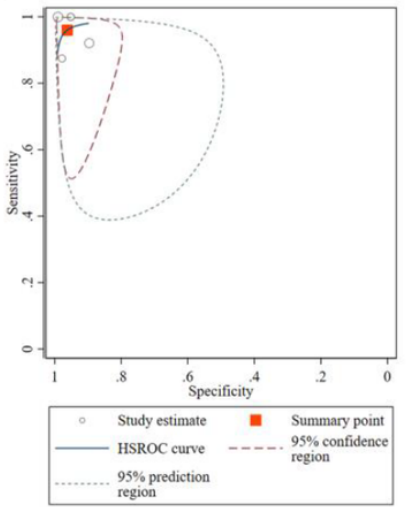

Figure 2

The HSROC plots of TE, 2D-SWE and MRE for the sensitivity and specificity in fibrosis stage $F \geq 2$ and $F=4$. The study estimate represents the data of each included study and the size of the circle represents the weight of each study based on the number of patients; the summary point represents the summary sensitivity and specificity; the $95 \%$ confidence region represents the $95 \%$ confidence interval of the summary sensitivity and specificity; the $95 \%$ prediction region represents $95 \%$ confidence interval of sensitivity and specificity of each individual study included in the meta-analysis. (A) HSROC plot of TEfor fibrosis stage $F \geq 2$, (B) HSROC plot of 2D-SWE for fibrosis stage $F \geq 2$, (C) HSROC plot of MRE for fibrosis stage $F \geq 2$, (D) HSROC plot of TE for fibrosis stage $F=4$. (E) HSROC plot of 2D-SWE for fibrosis stage $F=4$. (F) HSROC plot of MRE for fibrosis stage $F=4.150 \times 150 \mathrm{~mm}(300 \times 300 \mathrm{DPI})$ 


\begin{tabular}{|c|c|c|c|}
\hline \multicolumn{2}{|l|}{$\begin{array}{l}\text { Study } \\
\text { ID }\end{array}$} & \multirow{2}{*}{$\begin{array}{l}\text { DOR }(95 \% \mathrm{Cl}) \\
15.63(6.46,37.84)\end{array}$} & \multirow{2}{*}{$\begin{array}{l}\% \\
\text { Weight } \\
5.94\end{array}$} \\
\hline Zhang2015 & $\rightarrow$ & & \\
\hline Leung2013 & & $15.17(7.80,29.52) 7$ & 7.98 \\
\hline Yao2020 & $\rightarrow-$ & $2.91(0.96,8.82) \quad 4$ & 4.44 \\
\hline Zeng2017 & & $16.20(8.77,29.92) 8$ & 8.56 \\
\hline Liu2014 & & $12.21(4.19,35.56) \quad 4$ & 4.67 \\
\hline Li2018 & & $23.29(10.62,51.08) 6$ & 6.78 \\
\hline Gaia2011 & $\rightarrow$ & $4.38(1.59,12.08) \quad 5$ & 5.00 \\
\hline Seo2015 & $\rightarrow$ & $7.00(4.63,10.57) \quad 1$ & 11.05 \\
\hline Cardoso2011 & $\rightarrow$ & $21.07(10.05,44.15) 7$ & 7.22 \\
\hline Osakabe 2011 & & $34.84(1.83,664.91) 0$ & 0.85 \\
\hline Cho 2011 & $\rightarrow-$ & $16.34(5.89,45.32) 4$ & 4.97 \\
\hline Kim BK2012 & & $23.11(7.57,70.53) 4$ & 4.40 \\
\hline Kim US2012 & $\rightarrow-$ & $14.91(5.34,41.63) 4$ & 4.92 \\
\hline Kumar 2013 & $\rightarrow$ & $12.29(5.99,25.22) 7$ & 7.42 \\
\hline Zhao 2017 & $\rightarrow$ & $6.18(1.98,19.24) \quad 4$ & 4.29 \\
\hline Shen 2019 & $\rightarrow$ & $8.05(5.53,11.71) \quad 1$ & 11.52 \\
\hline Overall (I-squared $=49.5 \%, \mathrm{p}=0.013$ ) & & $11.59(8.75,15.33) \quad 1$ & 100.00 \\
\hline \multicolumn{2}{|c|}{$\begin{array}{l}\text { NOTE: Weights are from random effects analysis! } \\
\text { B Study }\end{array}$} & & $\%$ \\
\hline ID & & DOR $(95 \% \mathrm{Cl})$ & Weight \\
\hline Park2019 & & $11.45(3.25,40.39)$ & 12.06 \\
\hline Yao2020 & & $12.25(3.40,44.20)$ & 11.72 \\
\hline Zeng2017 & & $25.94(12.93,52.05)$ & 26.40 \\
\hline \multicolumn{2}{|l|}{ Leung2013 } & $764.93(26.38,159.83)$ & 19.52 \\
\hline \multicolumn{2}{|l|}{ Liu2019 } & $34.83(11.00,110.30)$ & 13.82 \\
\hline \multicolumn{2}{|l|}{ Zeng2014 } & $23.00(8.28,63.89)$ & 16.47 \\
\hline \multicolumn{2}{|c|}{ Overall (I-squared $=30.5 \%, p=0.207$ ) } & $26.30(16.03,43.13)$ & 100.00 \\
\hline \multicolumn{2}{|c|}{ NOTE: Weights are from random effects analysis } & & \\
\hline \multicolumn{2}{|c|}{ C Study } & & $\%$ \\
\hline \multicolumn{2}{|l|}{ ID } & DOR $(95 \% \mathrm{CI})$ & Weight \\
\hline \multicolumn{2}{|l|}{ Park2019 } & $81.00(9.39,698.49)$ & 23.20 \\
\hline \multicolumn{2}{|l|}{ Shi2014 } & $327.75(57.36,1872.81)$ & 35.45 \\
\hline \multicolumn{2}{|l|}{ Venkatesh2014 } & $1257.67(49.23,32127.10)$ & 10.25 \\
\hline \multicolumn{2}{|l|}{ Hennedige2017 } & $1257.67(49.23,32127.10)$ & 10.25 \\
\hline \multicolumn{2}{|l|}{ Wu2015 } & $267.00(27.51,2591.35)$ & 20.85 \\
\hline \multicolumn{2}{|l|}{ Overall (I-squared $=0.0 \%, \mathrm{p}=0.565)$} & $299.18(105.99,844.50)$ & 100.00 \\
\hline
\end{tabular}

\section{Figure 3}

Forest plot of tests. (A) TE in detecting significant fibrosis. (B) 2D-SWE in detecting significant fibrosis. (C) MRE in detecting significant fibrosis. 150×300mm $(300 \times 300 \mathrm{DPI})$ 


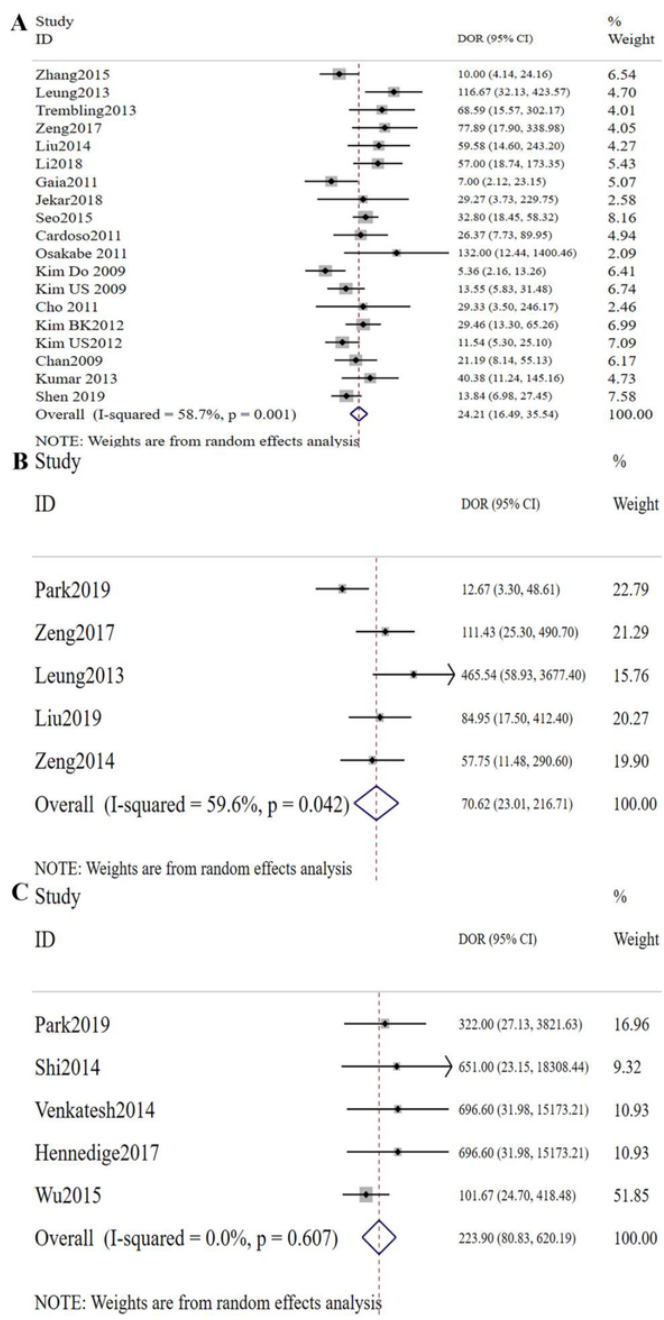

\section{Figure 4}

Forest plot of tests. (A) TE in detecting cirrhosis. (B) 2D-SWE in detecting cirrhosis. (C) MRE in detecting cirrhosis. 150×300mm (300×300 DPI)

\section{Supplementary Files}

This is a list of supplementary files associated with this preprint. Click to download.

- HBVmetasupplementarymaterial.docx 